\title{
Upgrades of the GANDALPH photodetachment detector towards the determination of the electron affinity of astatine
}

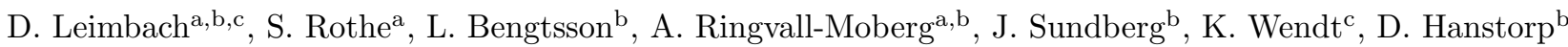 \\ ${ }^{a}$ CERN, Geneva, Switzerland \\ ${ }^{b}$ Department of Physics, University of Gothenburg, Gothenburg, Sweden \\ ${ }^{c}$ Institut für Physik, Johannes Gutenberg-Universität, Mainz, Germany
}

\begin{abstract}
The Gothenburg ANion Detector for Affinity measurements by Laser PHotodetachment (GANDALPH) has recently been built for measurements of electron affinities (EA) of radioisotopes. A first measurement campaign is aimed towards the determination of the EA of astatine, the rarest naturally occurring element on earth. In this work we present several upgrades of GANDALPH which have been implemented in order to facilitate EA measurements of radioisotopes where only low intensity ion beams $(<1 \mathrm{pA})$ can be produced.
\end{abstract}

Keywords: GANDALPH, negative ions, photodetachment, electron affinity

2010 MSC: 00-01, 99-00

\section{Introduction}

Negative ions are atoms or molecules with an additional electron, so that their net charge is negative. The binding potential of the additional electron in a negative 5 ion is mainly determined by inter-electronic interactions, making negative ions compelling systems for tests of theoretical models that go beyond the independent particle model [1. Due to the lack of bound states, the binding energy of the additional electron in a negative ion, called 10 electron affinity (EA), is the only atomic parameter which can be determined with high precision. The most common way to determine the electron affinity is by laser photodetachment threshold spectroscopy where a wavelength tunable laser beam is superimposed on a beam of negative ions

15 2. The electron affinity is obtained by determining the threshold for the onset of the photodetachment process.

\section{The GANDALPH beamline}

The Gothenburg ANion Detector for Laser PHotodetachment (GANDALPH) is a detector designed for measurements of electron affinities (EA) of radioisotopes by laser photodetachment threshold spectroscopy [3]. In GANDALPH, a continuous ion beam is merged with a pulsed laser beam over a $50 \mathrm{~cm}$ long path, as shown schematically in Fig. 1. The collinear geometry has the advantage of a 25 large interaction volume as well as a compressed Doppler spread 4. The ion beam enters the beamline with an angle of $10^{\circ}$ with respect to the central axis and is deflected into the main chamber. The beam then passes a steering box and is aligned to pass through two $6 \mathrm{~mm}$ diameter
30 apertures defining the interaction region where it is overalapped with a laser beam. While residual charged particles are deflected into a Faraday cup, neutrals proceed in their direction of flight into a neutral particle detector, as shown in Fig. 4. The neutral particle detector consists of a target box where neutrals impinge on a conductive and optically transparent target. Secondary electrons resulting from the impact are detected with a secondary electron multiplier (SEM). In order to distinguish between photoelectrons and electrons resulting from neutral particle impact, the data acquisition is triggered a few microseconds after the laser pulse, corresponding to the difference in time of flight of ions and photons. Further details of the GANDALPH detector are described in 3 .

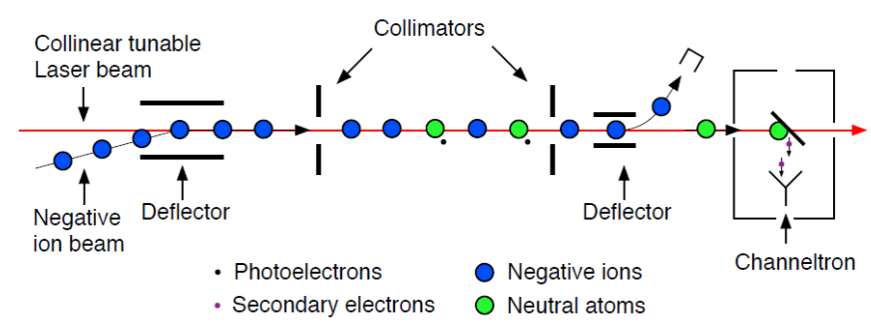

Figure 1: Schematic depiction of the GANDALPH beamline. The incoming negative ions (blue) are merged with the laser beam (red arrow) by two electrostatic deflector plates. Neutral atoms produced in the photodetachment process (green) proceed straight into the neutral particle detector where they impinge on a target and create secondary electrons which are detected with a SEM. Residual negative ions are bent into a Faraday cup.

A major goal with the GANDALPH detector is to de- 


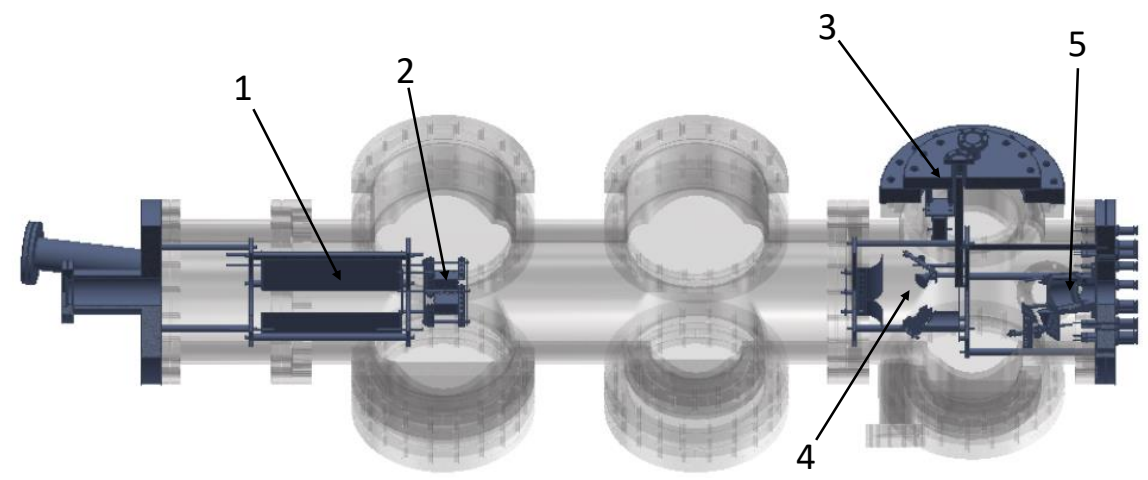

Figure 2: CAD-model of the upgraded GANDALPH beamline. 1) Deflector plates to bend the incoming ions into the chamber. 2) Steering box with aperture. 3) Silicon detector: Neutral atoms are implanted onto a moveable plate which is then retracted in front of the detector. 4) Faraday cup and SEM for beam tuning and background measurements. 5) Neutral particle detector.

rally occurring element on earth [6]. Astatine is of medical interest since its isotope ${ }^{211}$ At is a potential agent for tar- 85 geted alpha therapy [7, 8]. The EA of astatine, together with the previously measured first ionization potential (IP) 9 gives valuable benchmarks for quantum chemical calculations predicting the chemical properties of this element and its compounds [10, 11, 12] .

As a proof-of-principle towards the astatine experiment, the EA of ${ }^{128}$ I was determined at CERN-ISOLDE, marking the first ever photodetachment experiment of a radioactive isotope [3. During this initial campaign it became evident that the astatine experiment required significant improved experimental conditions, taking into account the low production rates of about 6000 ions $/ \mathrm{s}_{\text {of }} \mathrm{At}^{-}$

60 at ISOLDE. Hence, several upgrades of the beamline were necessary, which are described in the following.

\section{Upgrades of the GANDALPH detector}

Improving beam tuning capabilities. In order to facilitate ion beam tuning with low beam intensities through GAN-

65 DALPH, several upgrades were conceived and implemented. As a first step towards improved beam tuning, the apertures of the setup were upgraded. A traditional aperture for ion beam alignment has only a single circular hole. An indication of a possible ion beam misalignment is achieved 70 by measuring the ion current on the aperture plate. However, this does not give information about the direction ${ }^{95}$ of a possible misalignment. The apertures were therefore segmented into four separate pieces that can be read out individually. By measuring the current individually on all 75 four aperture plates, it is then possible to determine the beam position relative to the center of the aperture. For ${ }^{10}$ this purpose, a 4-channel ammeter with picoampere resolution was designed which is shown in Fig. 3. This ammeter was implemented on a printed circuit board (pcb) using

so four ultra-low input bias current operational amplifiers (op am, AD549, see [13]) in a feedback ammeter design [14. ${ }^{105}$ Since the currents are of the order of a few picoampere, great care must be taken to isolate the op amps inputs on the pcb. This is achieved by routing proper guard copper patterns around the signal inputs [15].

All ion beam optic plates in GANDALPH are controlled by high voltage (HV) supplies from Wenzel Elektronik (N1130 NIM modules). Since about 20 electrodes are required, a 24-channel voltage control instrument was designed to facilitate remote control of the HV supplies [16. Also, a LabVIEW GUI was developed for user-friendly operation of the instrument. Each channel has a resolution of 12 bits and a confirmed noise level corresponding to less than $1 \mathrm{mV}$.

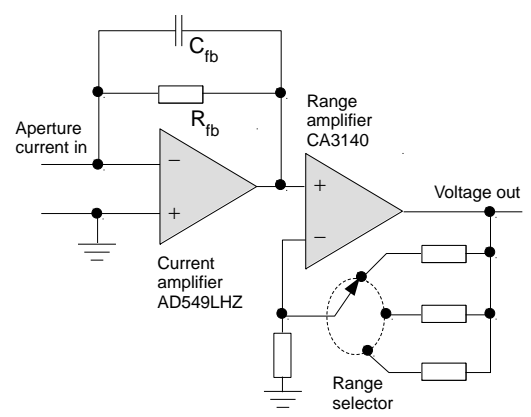

Figure 3: The current-to-voltage amplifier is implemented by an ultra-low bias current op amp, succeeded by a conventional op amp for voltage amplification and range selection.

Previously, initial beam tuning of low intensity radioactive beams was performed by steering the ion beam directly into the neutral particle detector, resulting in an increased background signal during the actual measurement. In order to circumvent this problem, an additional SEM dedicated for beam tuning and background measurements was installed in the neutral particle detector section. The electrostatic elements for bending the ion beam after the interaction was redesigned so it can either bend the beam into the Faraday cup (high intensity beam) or the SEM (low intensity beam). The redesign was performed using Autodesk Inventor Professional 2017 for the mechanical design and CST Studio for the simulation of the ion beam trajectories. A 3D representation of the new neutral 
particle detector flange is shown in Fig. 4. Additionally, 110 the previously used target, an ITO coated glass plate, was replaced with a graphene-coated glass plate, thereby im-130 proving laser transmission and background noise in the detector, as described in [17.

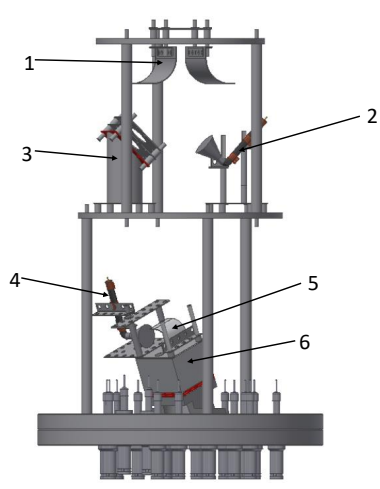

Figure 4: 3D model of the neutral particle detector flange. 1) ion beam deflectors 2) secondary electron multiplier 3) Faraday cup 4) secondary electron multiplier 5) electron bend 6) neutral particle detector box with graphene coated glass plate. The detector was assembled using self-produced as well as Kimball-physics eV parts [18.

Alpha spectroscopy setup. Specifically for the purpose of the measurement of alpha emitting particles such as astatine and polonium, an Ortec Alpha Aria spectrometer with a BU-024-600-AS detector was added to the GANDALPH detector. In order to avoid stray light and to be able to detect only the alpha decay coming from neutralized atoms, the silicon detector was mounted upstream the neutral particle detector, as shown in Fig. 2. The detector ${ }_{155}$ itself, shown in more detail in Fig. 5 is held by two metal plates, while signal and voltage bias are supplied via an SMA feedthrough.
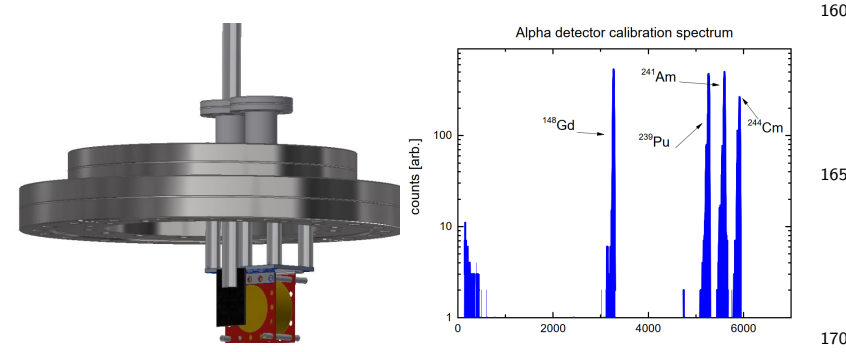

Figure 5: Left: 3D model of the alpha detector mount. The alpha particles are implanted on the aluminium plate (black) which is then retracted from the beam path to the alpha particle detector (gold) held sandwiched between two mounting plates (red). The signal and175 voltage bias is delivered via an SMA feedthrough. Right: Calibration spectrum performed with a source containing $\mathrm{Am}, \mathrm{Gd}, \mathrm{Pu}$ and $\mathrm{Cm}$.

A pneumatic feedthrough is used to descend an at- ${ }^{180}$ tached aluminium plate into the beam path of the neutral particles in front of the target box, such that only the detached atoms are implanted. After a set implantation time the plate is retracted in front of the alpha detector where a spectrum of the decay products is obtained. Such a spectrum of the alpha detector, obtained during calibration, is shown in Fig. 5 with a resolution of about $19 \mathrm{keV}$ (FWHM). Data acquisition and timing is performed using a self developed LabView software.

\section{Conclusion}

We conclude that the GANDALPH detector has successfully undergone several upgrades improving the ion beam tuning and detection for photodetachment experiments of radioisotopes. Segmented apertures as well as a 404 channel ammmeter with picoamp resolution and an additional SEM for tuning and background measurements were introduced. Additionally, a new control system for the beam optics was put in place using a 24 Channel voltage control instrument and a LabView GUI. An alpha detection setup was integrated into the GANDALPH detector and tested with a radioactive source.

It should finally be mentioned that the GANDALPH detector with the described upgrades was successfully used to determine the EA of astatine at CERN-ISOLDE in October 2018 [19.

\section{Acknowledgements}

This work is supported by the Swedish Research Council.

\section{References}

[1] D. J. Pegg, Structure and dynamics of negative ions, Reports on Progress in Physics 67 (2004) 857.

[2] T. Andersen, Atomic negative ions: structure, dynamics and collisions, Physics Reports 394 (2004) $157-313$.

[3] S. Rothe, et al., Laser photodetachment of radioactive 128 I-, Journal of Physics G: Nuclear and Particle Physics 44 (2017) 104003.

[4] S. Kaufman, High-resolution laser spectroscopy in fast beams, Optics Communications 17 (1976) $309-312$.

[5] S. Rothe, et al., Determination of the electron affinity of astatine and polonium by laser photodetachment, Technical Report CERN-INTC-2016-017. INTC-P-462, CERN, Geneva, 2016. URL: https://cds.cern.ch/record/2120161.

[6] I. Asimov, The natural occurrence of short-lived radioisotopes, J. Chem. Educ. 30 (1953) 616.

[7] M. Zalutsky, et al., Astatine-211: production and availability., Current radiopharmaceuticals 4 (2011) 177-85.

[8] D. S. Wilbur, Enigmatic astatine, Nature Chemistry 5 (2013) 246-246.

[9] S. Rothe, et al., Measurement of the first ionization potential of astatine by laser ionization spectroscopy, Nature Communications 4 (2013) 1835.

[10] A. Borschevsky, L. F. Pašteka, V. Pershina, E. Eliav, U. Kaldor, Ionization potentials and electron affinities of the superheavy elements 115-117 and their sixth-row homologues $\mathrm{Bi}, \mathrm{Po}$, and at, Physical Review A - Atomic, Molecular, and Optical Physics 91 (2015) 1-5. 
[11] Z. Chang, J. Li, C. Dong, Ionization potentials, electron affinities, resonance excitation energies, oscillator strengths, and ionic radii of element uus $(\mathrm{z}=117)$ and astatine, The Journal of Physical Chemistry A 114 (2010) 13388-13394. PMID: 21141866.

[12] J. Champion, C. Alliot, E. Renault, B. M. Mokili, M. Chérel, N. Galland, G. Montavon, Astatine Standard Redox Potentials and Speciation in Acidic Medium, Journal of Physical Chemistry A 114 (2010) 576-582.

[13] Analog Devices, Ultralow Input Bias Current Operational Amplifier, Rev. H, Analog Devices, Inc, Norwood, Maine, 2008.

[14] Keithley Instruments Inc., Low Level Measurements Handbook, 2004.

15] Analog Devices, Monolithic Precision, Low Power FET-Inut Electrometer Op Amp, Analog Devices Inc., 1997.

[16] L. Bengtsson, 24-channel dual microcontroller-based voltage controller for ion optics remote control, Nuclear Instruments and Methods in Physics Research A 890 (2018) 96-101.

200 [17] J. Warbinek, et al., A graphene-based neutral particle detector, Applied Physics Letters (2018). Submitted.

[18] Kimball physics, eV Parts general information and construction techniques, Kimball Physics, Inc., 2009.

[19] D. Leimbach, et al., Determination of the electron affinity of astatine (2018). In preparation. 